\title{
Pushing the hyperpolarizability to the limit
}

\author{
Juefei Zhou, Mark G. Kuzyk, and David S. Watkins* \\ Department of Physics and Astronomy; and Mathematics*, \\ Washington State University, Pullman, Washington 99164-2814
}

(Dated: April 2, 2018)

\begin{abstract}
We use numerical optimization to find a one-dimensional potential energy function that yields the largest hyperpolarizability, which we find is within $30 \%$ of the fundamental limit. Our results reveal insights into the character of the potential energy functions and wavefunctions that lead to the largest hyperpolarizability. We suggest that donor-acceptor molecules with a conjugated bridge with many sites of reduced conjugation to impart conjugation modulation may be the best paradigm for making materials with huge hyperpolarizabilities that approach the fundamental limit.
\end{abstract}

The study of nonlinear optical materials has been driven by the quest for making the largest possible nonlinear response in a medium for use in a broad range of optical applications such as telecommunications, 1] optical data storage, 2] threedimensional nano-photolithography, [3, 44 and making new materials [5] for novel cancer therapies. [6]

A natural questions arises whether there is a limit to the nonlinear response and if so, how does one make a molecule to get to the limit. The former has been answered in the affirmative using sum rules, 7, 8, 9, 10, 11, 12. and the fundamental limit is given by,

$$
\beta_{M A X}=\sqrt[4]{3}\left(\frac{e \hbar}{\sqrt{m}}\right)^{3} \cdot \frac{N^{3 / 2}}{E_{10}^{7 / 2}}
$$

where $N$ is the number of electrons and $E_{10}$ the energy to the first excited state. This result has not yielded clear guidance on how to design better molecules, but rather has set the bar for assessing the performance of molecules. In fact, the largest nonlinear susceptibilities of the best molecules fall short of the fundamental limit by a factor of $10^{3 / 2}$. 11, 13, 14] A Sum-Over-States (SOS) calculation of the hyperpolarizability [15] using the analytical wavefunctions of the clipped harmonic oscillator yields a value that is within a factor of 2 of the limit. 14. Thus, the factor-of-thirty gap between the fundamental limit and the apparent upper bound of the best molecules is not of a fundamental nature. So, it should be possible in principle to make materials with second-order susceptibilities that are a factor of 30 bigger than the currently best materials. In this letter, we report on the character of the potential energy function of a one-dimensional system that yields a hyperpolarizability, $\beta$, that is within $30 \%$ of the fundamental limit, and use this potential to propose a new paradigm for molecular engineering.

There are two equivalent expressions for $\beta$. The standard one, $\beta_{S O S}$, as calculated by Orr and Ward, 15] and the dipole-free expression, $\beta_{D F}$. [16] The standard one is overspecified in the sense that it is possible to pick unphysical values of the energies and matrix elements, which violate the precepts of quantum mechanics. The dipole free expression, in contrast, is simplified into a reduced form that contains no dipole terms. Since the two expressions should yield the same results if all the matrix

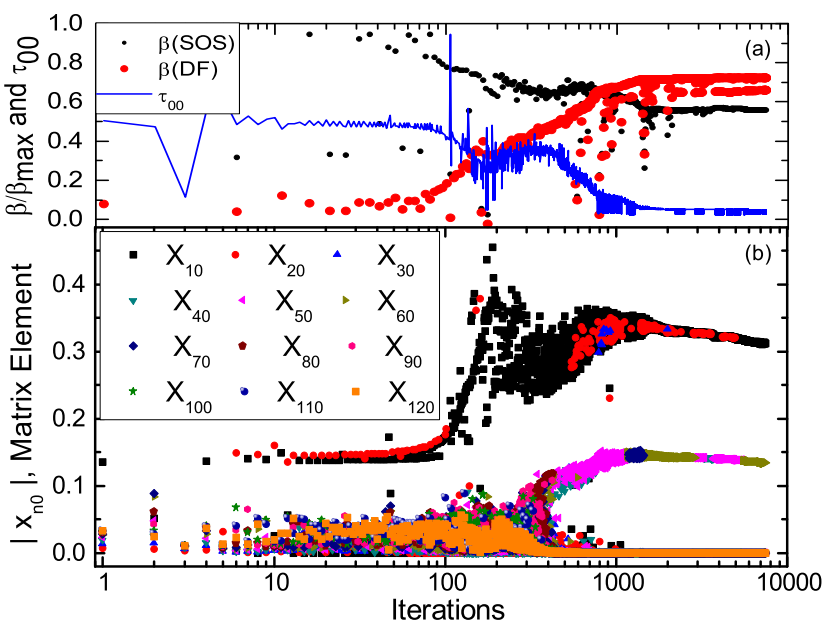

FIG. 1: Evolution of (a) $\beta$ and $\tau_{00}$; and (b) $x_{n 0}$ as a function of number of iterations of optimization.

elements are consistent with the sum rules, deviations between them can be used as a convergence test. [16] However, we later discuss how to treat exceptions where $\beta_{D F}$ is accurate and $\beta_{S O S}$ is not.

Our approach is to numerically calculate $\beta$ for a specific potential, then, to use an optimization algorithm that continuously varies the potential in a way that maximizes $\beta$ - using the convergence test to determine if the result is reliable. Our code is written in MATLAB. For each trial potential we use a quadratic finite element method [17 to approximate the Schrödinger eigenvalue problem and the implicitly restarted Arnoldi method [18] to compute the wave functions and energy levels. To optimize $\beta$ we use the Nelder-Mead simplex algorithm [19.

Figure 1. shows the evolution of the hyperpolarizability as a function of the number of iterations of the optimization algorithm, applied to $\beta_{D F}$. All hyperpolarizabilities are normalized to the fundamental limit. The initial potential energy function is a hyperbolic tangent with infinite potential at the boundaries. We select this potential because it is localized near the origin, yet flat elsewhere, allowing the optimization process to work without any initial biases. Also, it meets the criteria of 
being noncentrosymmetric, as required for nonvanishing hyperpolarizability. After 7,000 iterations, the algorithm converges to just over $\beta_{D F} / \beta_{M A X}=0.72$, the largest hyperpolarizability seen to date. No other starting potential, including polynomials, and fractional exponents, leads to larger $\beta_{D F}$.

As a second convergence test, we use the fractional deviation from the ground state sum rule, which in terms of $\kappa_{00}$ as defined in the literature, is given by, 20]

$$
\tau_{00}=1-\sum_{n} \frac{E_{n 0}}{E_{10}}\left|\frac{x_{n 0}}{x_{10}^{\max }}\right|^{2} \equiv 1-\kappa_{00},
$$

where $E_{n 0}$ is the energy difference between state $n$ and $0, x_{n m}$ is the position matrix element between state $n$ and $m$, and $x_{10}^{\max }$ is the fundamental limit of $x_{10} \cdot \tau_{00}$ approaches zero when the optimization process yields maximum $\beta_{D F}$, supporting the fact that the numerical computations accurately represent the system. When, on the other hand, $\beta_{S O S}$ is optimized, $\tau_{00}$ is large and the two forms of $\beta$ diverge appreciably, illustrating the robustness of the dipole-free form and the pitfalls of using $\beta_{S O S}$ for optimization. Our calculations of $\beta$ include a total of 15 states, so a large value of $\tau_{00}$ is more of an indication that not enough states are being used in $\beta_{S O S}$ rather than inaccuracies of the wave functions. The fact that $\beta_{S O S}$ and $\beta_{D F}$ do not converge illustrates the need for two independent convergence tests.

Figure 10 shows the evolution of the position matrix elements. When $\beta_{D F}$ is small, many of these matrix elements are non-negligible, so many states will contribute to $\beta$. By a thousand iterations when the hyperpolarizability approaches its largest value, all matrix elements vanish except for two of them. This clearly shows that as the fundamental limit of $\beta$ is approached, the system collapses to a three-level model for $\beta$. This is consistent with the three-level ansatz, previously proposed, that a three-level model describes a system with a hyperpolarizability at the fundamental limit. 21] There is mounting evidence that the three-level ansatz also is obeyed in planar molecules. 22 We stress that our results do not prove the converse; that all molecules with hyperpolarizabilities at the fundamental limit must be represented by a three-level model. However, since (1) the threelevel ansatz is used to determine the fundamental limits, and, all reliable measurements and calculations yield hyperpolarizabilities below the fundamental limit; and (2) numerical optimization yields a three-level model, this form of induction provides strong support for the ansatz.

Figure 2 shows the evolution of the potential energy function for a length scale on the order of a large molecule. Optimization of the hyperpolarizability clearly favors a potential with large oscillations. Interestingly, all starting potentials we have studied, independent of their initial form - such as polynomials, power laws, and piecewise continuous functions, develop such wild oscillations of about the same period when the hyperpolarizability is maximized. We find that these oscillations serve to localize each eigenfunction at different positions. Early in the

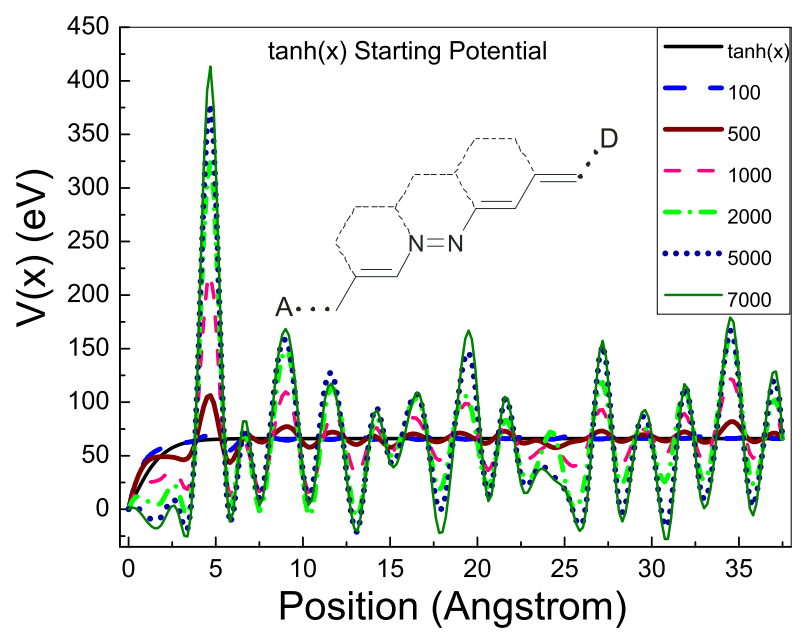

FIG. 2: Evolution of potential energy as a function iteration. Inset shows a class of proposed molecules, where "..." refers to a continuation of the bridge using the same theme.

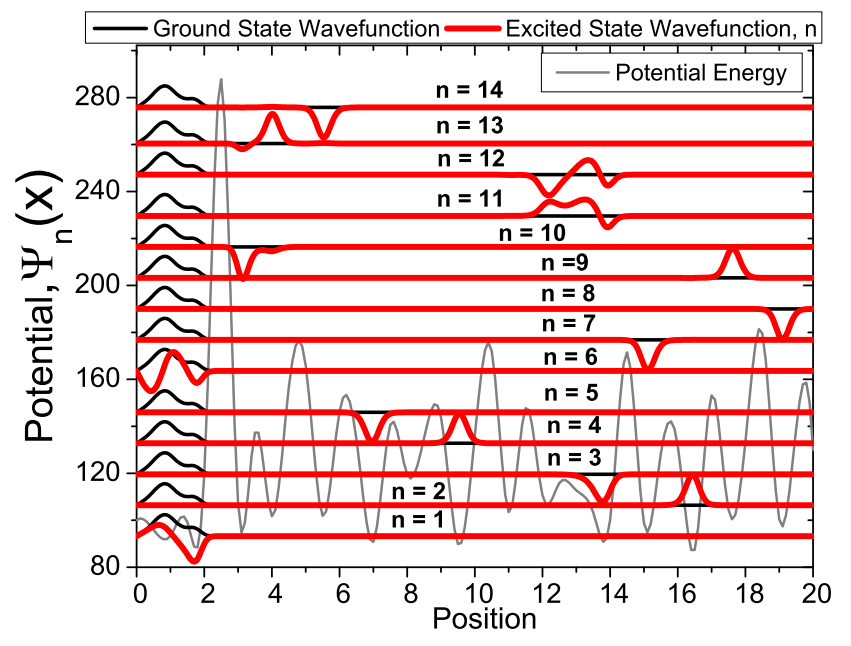

FIG. 3: Potential energy and energy eigenfunctions after 7,000 iterations, when $\beta_{D F}$ is optimized.

optimization process, the wavefunctions are all delocalized while at 7,000 iterations, most of the wavefunctions are mutually non-overlapping.

Figure 3 shows the eigenfunctions and potential energy function after 7,000 iterations. Only excited states number 1 and 6 have appreciable overlap with the ground state and with each other. As such, the only term that contributes to the hyperpolarizability is proportional to $x_{01} x_{16} x_{60} / E_{10} E_{60}$. Note that with other starting potentials, states other then $0,1,6$ may be important. Most significantly, $\beta$ is maximum for a three-level system.

Under the three-level ansatz, the normalized hyperpolarizability can be expressed as a product of two functions, $\beta / \beta_{M A X} f(E) G(X),[14]$ where $X=x_{10} / x_{10}^{M A X}$ and $E=E_{10} / E_{20}$. "1" and "2" label the states with the largest transition moments to the ground state. The function $f(E)$ is maximum when $E=0(f(0)=1)$ and 
$G(X)$ is maximum when $X=\sqrt[-4]{3}(G(\sqrt[-4]{3})=1)$. So, a hyperpolarizability that approaches the fundamental limit should have two dominant states that have well separated eigenenergies; and, $x_{10} \sqrt[-4]{3} x_{10}^{M A X}$. The optimized wavefunctions yield $E 0.314$ and $X=0.775$, so $f(E)=0.892$ and $G(X)=0.999$. The transition moment is near optimal but the energy level spacing can be improved. If the system were truly 3 levels, $\beta / \beta_{\text {MAX }}=f(E) G(X)=0.89$ compared with the optimized value of $\beta_{D F} / \beta_{M A X}=0.72$.

One might argue that our results are artificial because we are using only 15 states, and, because our space is bounded by an infinite potential. These two issues go hand in hand. In our calculations, the potential energy function is represented by a cubic spline of 20 points. As such, it would be numerically impossible to localize more than about 15 eigenfunctions. Furthermore, higherenergy wavefunctions would interact with the walls; and, for high-enough energies, the wavefunctions would have the character of a particle in a box. So, to increase the number of energy levels, one would need to increase the number of points in the spline, which would lead to more oscillations that would localize more of the excited states. Based on the pattern that we have observed, when increasing the number of states and making the potential correspondingly broader, we expect that more non-overlapping wavefunctions would develop, yielding a similar conclusion. So, in the limit of infinitely wide space, the conclusion should be the same.

In summary, we have applied an optimization process to determine what potential energy function yields the largest value of the hyperpolarizability. As a function of iteration, the hyperpolarizability approaches the fundamental limit, but never exceeds it. When $\beta$ converges to the maximal value, the expression for $\beta$ collapses to a three-level model, supporting the three-level ansatz.
Strong oscillations in the potential energy function serve to isolate the wavefunctions to prevent overlap between all states but three of them. This behavior suggests that long linear organic molecules with regions of conjugation (representing the dips in the potential) interspersed by regions of reduced conjugation (representing the peaks) and flanked by a donor and acceptor to break the symmetry, would be a promising new paradigm.

The inset in Figure 2 shows one example of a new molecular paradigm that should exhibit the required modulation of conjugation over a single and double bond pair spatial period. The dashed bonds represent the absence or presence of rings to control the degree of conjugation and/or molecular planarity. Note that at 100 iterations, the oscillations are on the order of $1 \mathrm{eV}$ (common energy scale for molecules), where $\beta / \beta_{M A X} 0.2$ - well above the apparent limit of $\beta / \beta_{M A X}=0.03$. So, our new paradigm should lead to a tenfold improvement over today's best molecules. Alternatively, our method could guide the fabrication of multiple quantum well structures using a variety of layered organic molecules that impart to it an oscillating potential energy profile.

We plan to apply our general method to higher-order nonlinear-optical susceptibilities. In addition, it can be applied to resonant processes and to any general combinations of laser wavelengths. With regards to the second hyperpolarizability, $\gamma$, since past calculations suggest that the three-level ansatz also should hold, we would expect the same sort of conclusion. Should this approach be successful, new and exceptional materials could be developed to make more efficient devices and novel applications.

Acknowledgements: MGK thanks the National Science Foundation (ECS-0354736) and Wright Paterson Air Force Base for generously supporting this work.
[1] Q. Y. Chen, L. Kuang, Z. Y. Wang, and E. H. Sargent, Nano. Lett. 4, 1673 (2004).

[2] C. E. Olson, M. J. R. Previte, and J. T. Fourkas, Nature Materials 1, 225 (2002).

[3] B. H. Cumpston, S. P. Ananthavel, S. Barlow, D. L. Dyer, J. E. Ehrlich, L. L. Erskine, A. A. Heikal, S. M. Kuebler, I.-Y. S. Lee, D. McCord-Maughon, J. Qin, H. Röckel, M. Rumi, X.-L. Wu, S. R. Marder, and J. W. Perry, Nature 398, 51 (1999).

[4] S. Kawata, H.-B. Sun, T. Tanaka, and K. Takada, Nature 412, 697 (2001).

[5] A. Karotki, M. Drobizhev, Y. Dzenis, P. N. Taylor, H. L. Anderson, and A. Rebane, Phys. Chem. Chem. Phys. 6, 7 (2004).

[6] I. Roy, O. T. Y., H. E. Pudavar, E. J. Bergey, A. R. Oseroff, J. Morgan, T. J. Dougherty, and P. N. Prasad, J. Am. Chem. Soc. 125, 7860 (2003).

[7] M. G. Kuzyk, Opt. Lett. 25, 1183 (2000).

[8] M. G. Kuzyk, IEEE Journal on Selected Topics in Quantum Electronics 7, 774 (2001).
[9] M. G. Kuzyk, Phys. Rev. Lett. 85, 1218 (2000).

[10] M. G. Kuzyk, Opt. Lett. 28, 135 (2003).

[11] M. G. Kuzyk, Phys. Rev. Lett. 90, 039902 (2003).

[12] M. G. Kuzyk, J. Nonl. Opt. Phys. \& Mat. 13, 461 (2004).

[13] M. G. Kuzyk, Optics \& Photonics News 14, 26 (2003).

[14] K. Tripathi, J. Pérez Moreno, M. G. Kuzyk, B. J. Coe, K. Clays, and A. M. Kelley, J. Chem. Phys. 121, 7932 (2004).

[15] B. J. Orr and J. F. Ward, Molecular Physics 20, 513 (1971).

[16] M. G. Kuzyk, Phys. Rev. A 72, 053819 (2005).

[17] O. C. Zienkiewicz, R. L. Taylor, and J. Z. Zhu, The Finite Element Method: Its Basis and Fundamentals (Butterworth-Heinemanm, 2005), 6th ed.

[18] D. C. Sorensen, SIAM J. Matrix Anal. Appl. 13, 357 (1992).

[19] J. C. Lagarias, J. A. Reeds, M. H. Wright, and P. Wright, SIAM J. Optim. 9, 112 (1998).

[20] M. G. Kuzyk, J. Nonl. Opt. Phys. \& Mat. 15, 77 (2006).

[21] M. G. Kuzyk, Phys. Rev. Lett. 95, 109402 (2005). 
[22] M. G. Kuzyk and D. S. Watkins, arXiv:physics/0601172 (2006). 